\title{
High Efficiency RF Power Amplifier Designed With Harmonic Real-Time Active Load-Pull
}

\author{
Xian Cui, Seok Joo Doo, Patrick Roblin, Member, IEEE, Jeffrey Strahler, Member, IEEE, and \\ Roberto G. Rojas-Teran, Fellow, IEEE
}

\begin{abstract}
In this work, a high efficiency p-HEMT radio frequency power amplifier $(P A)$ is designed using a new multiharmonic real-time active load-pull using the large signal network analyzer. This technique synthesizes a large set of instantaneous load mismatches to quickly find the optimal harmonic impedances, so as to achieve high PA efficiency in a shortened design cycle. At $2 \mathrm{GHz}$ a demo power amplifier implemented with a p-HEMT demonstrated a power added efficiency (PAE) of $68.5 \%$ for 18.0 dBm output power, while achieving a maximum PAE of $75 \%$ below the $1 \mathrm{~dB}$ compression point for $18.6 \mathrm{dBm}$ output power.
\end{abstract}

Index Terms-Large signal network analyzer (LSNA), power added efficiency (PAE), power amplifier (PA), real-time active load-pull (RT-ALP).

\section{INTRODUCTION}

$\mathbf{N}$ OWADAYS the availability of high efficiency radio frequency (RF) power amplifiers (PA) is a critical factor for wireless mobile devices to extend battery operation time as well as reduce the heat sink form factor and weight. Nonlinear power amplifiers, such as Class F [1], [2], achieve high efficiency by minimizing the overlap between device voltage and current to reduce the power dissipation of the transistor itself. Conventional designs using circuit simulators are limited by the accuracy of nonlinear large signal models and device package parasitics [3]. Passive or active load-pull [4]-[6] can be used to determine the optimal load conditions for the power transistor but it is a time-consuming process, especially in nonlinear PA design where both the impedance amplitude and phase must be swept for each harmonic to find the optimal terminations.

A real-time active load-pull (RT-ALP) [7] based on the large signal network analyzer (LSNA) was introduced for ultra-fast impedance sweeping. It was extended to multiharmonics for nonlinear PA design in [8] and multiharmonic load conditions for CW operation of a GaN HEMT with PAE of $80 \%$ were extracted. This work indicated that RT-ALP offers great opportunities for speeding up the PA design.

This letter presents the first complete design of a $2 \mathrm{GHz}$ high efficiency p-HEMT PA using the new multiharmonic RT-ALP for drastically shortening the PA design cycle. Real-time tunings not only at the fundamental but also the second and third harmonic frequencies are utilized to quasi-interactively determine

Manuscript received October 17, 2007; revised January 8, 2008. This work was supported in part by the National Science Foundation under Grant ECS0622003.

The authors are with the Department of Electrical and Computer Engineering, The Ohio State University, Columbus, OH 43210 USA (e-mail: roblin@ece. osu).

Color versions of one or more of the figures in this letter are available online at http://ieeexplore.iee.org.

Digital Object Identifier 10.1109/LMWC.2008.918913

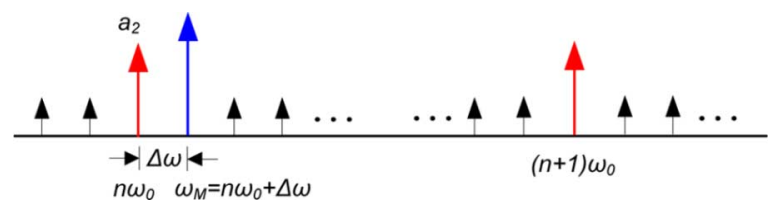

Fig. 1. Frequency domain representation of the incident wave $a_{2}$ actively injected (blue) at the transistor's output in RT-ALP measurements.

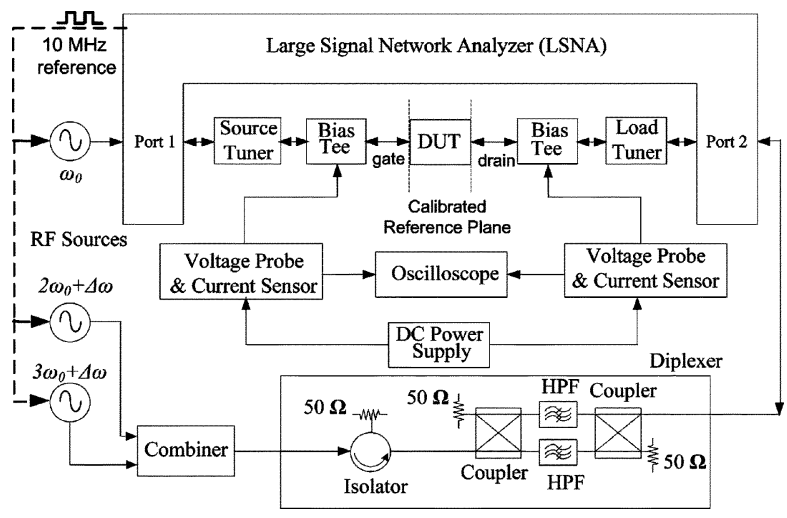

Fig. 2. Multiharmonic real-time active load-pull configuration.

the optimal load terminations. The goal is to select a load-line minimizing $\int_{0}^{T} i_{D}(t) \times v_{D S}(t) d t$ instead of $i_{D}(t) \times v_{D S}(t)$ for a given input power. The corresponding matching networks are then designed to realize the optimal multiharmonic reflection coefficients obtained. The constructed p-HEMT PA is then characterized and its performances are compared to the RT-ALP prediction.

\section{Multiharmonic RT-ALP Technique}

At the $n$-th harmonic frequency $\left(n \omega_{0}\right)$, the RT-ALP method applies at the output a CW tone $a_{2}\left(n \omega_{0}+\Delta \omega\right)$ with a frequency offset $\Delta \omega$ (Fig. 1) from the targeted $n \omega_{0}$ so that the phase of the harmonic load reflection coefficient is continuously swept in each LSNA measurement (e.g., $10 \mathrm{~ms}$ ). The reflection coefficient loci is changed by varying the power levels for $a_{2}\left(n \omega_{0}+\Delta \omega\right)$, producing a set of harmonic load reflection coefficient loci mapping the Smith chart (see Fig. 3 in Section III). Circles with larger radius are associated with larger injected power levels. The swept dynamic RF loadlines of the device are simultaneously obtained.

The configuration of the multiharmonic RT-ALP is shown in Fig. 2. The top RF source provides a $\mathrm{CW}$ signal at the fundamental frequency $\omega_{0}$ to drive the transistor input. The two bottom sources inject signals at $\left(2 \omega_{0}+\Delta \omega, 3 \omega_{0}\right)$ or 


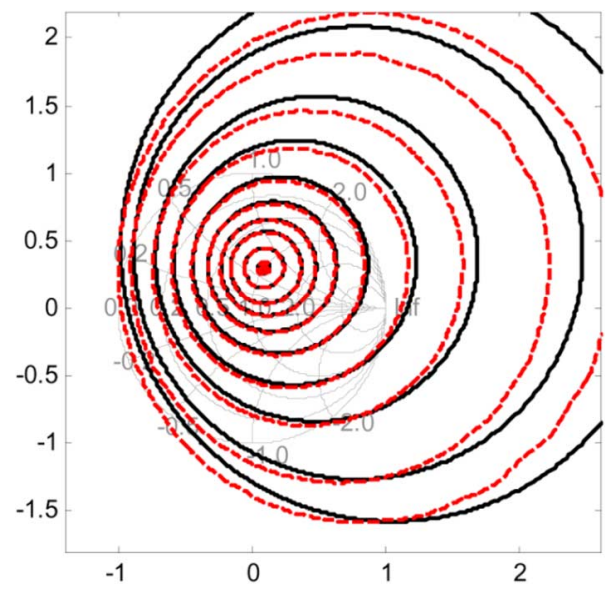

Fig. 3. Loci of $\Gamma_{L}\left(2 \omega_{0}, t\right)$ obtained from the $\left(2 \omega_{0}+\Delta \omega\right)$ real-time swept phase measurements by accounting for the two dominant tones (solid line) or all the sideband tones (dashed line) with (1). Each circle is measured in $10 \mathrm{~ms}$.

$\left(2 \omega_{0}, 3 \omega_{0}+\Delta \omega\right)$ in the RT-ALP case (swept phase measurement) or $\left(2 \omega_{0}, 3 \omega_{0}\right)$ in the constant phase measurement case. The bias voltage and current variations of the device are displayed on the oscilloscope. The diplexer provides a path for the injected harmonic signals to reach the transistor output while maintaining an impedance match for $\omega_{0}$ at port 2 .

The load reflection coefficient $\Gamma_{L}\left(n \omega_{0}, t\right)$ can be easily extracted from the RT-ALP data by either accounting for only the two dominant tones $\left(n \omega_{0}\right)$ and $\left(n \omega_{0}+\Delta \omega\right)$, or all the sideband tones with $S S B$ the total number of single side band modulation tones measured by the LSNA using

$$
\Gamma_{L}\left(n \omega_{0}, t\right)=\frac{\sum_{p=-S S B}^{S S B} a_{2}\left(n \omega_{0}+p \Delta \omega\right) e^{j p \Delta \omega t}}{\sum_{p=-S S B}^{S S B} b_{2}\left(n \omega_{0}+p \Delta \omega\right) e^{j p \Delta \omega t}} .
$$

The contours of output power and PAE can then also be plotted to select the optimal harmonic load conditions.

\section{PA Design With RT-ALP AND MEASUREMENT RESUlts}

The above multiharmonic RT-ALP methodology is applied to a packaged pHEMT (Agilent ATF-54143) biased around cut-off $\left(V_{D S}=30 \mathrm{~V}, V_{\mathrm{GS}}=0.31 \mathrm{~V}\right)$. In this study the LSNA acquired spectral data up to the fourth harmonic each with 99 tones $(S S B=49)$. The modulation frequency $\Delta f$ is $200 \mathrm{kHz}$.

With a RT-ALP first run at $f_{0}=2 \mathrm{GHz}$ with $6.5 \mathrm{dBm}$ input power, the optimal load at the fundamental is selected as $\Gamma_{L}\left(\omega_{0}, t\right)=-0.2$ for the subsequent $2 f_{0}$ and $3 f_{0}$ RT-ALP.

Fig. 3 shows the extracted $\Gamma_{L}\left(2 \omega_{0}, t\right)$ result from the second harmonic RT-ALP data at $4 \mathrm{GHz}$. The dashed line loci are obtained when accounting for all sideband tones in (1), and the solid line loci when considering only the two dominant tones. At low power levels (small radii) the latter is a good approximation to the former as expected. The PAE contours are shown in Fig. 4. As can be seen, the realistic second harmonic impedance with the highest PAE (case A) near the Smith chart's edge differs from the class B/F ideal short. This high PAE is verified with the constant phase measurement to be $71.92 \%$ with an output power of $60.8 \mathrm{~mW}$.

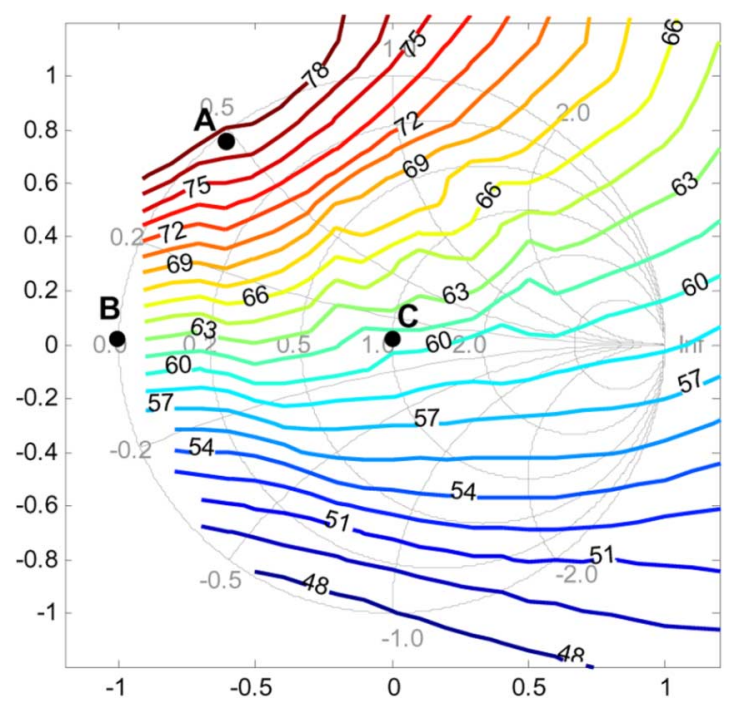

Fig. 4. PAE contour plot in the $\Gamma_{L}\left(2 \omega_{0}\right)$ plane, obtained from the $\left(2 \omega_{0}+\Delta \omega\right)$ real-time swept phase measurements (based on dashed line contour in Fig. 3).

TABLE I

CoMPARISON OF THREe TyPICAL CASES: CASE A, B, C

\begin{tabular}{ccccc}
\hline \multirow{2}{*}{$\Gamma_{\mathrm{L}}\left(2 \omega_{0}\right)$} & \multicolumn{2}{c}{ PAE (\%) } & \multicolumn{2}{c}{ Output Power (mW) } \\
& $\begin{array}{c}\text { Swept } \\
\text { Phase }\end{array}$ & $\begin{array}{c}\text { Constant } \\
\text { Phase }\end{array}$ & $\begin{array}{c}\text { Swept } \\
\text { Phase }\end{array}$ & $\begin{array}{c}\text { Constant } \\
\text { Phase }\end{array}$ \\
\hline $\begin{array}{c}\text { Case A: } \\
\text { (optimal 0.6+j0.7) }\end{array}$ & 77.04 & 71.92 & 60.26 & 60.80 \\
$\begin{array}{c}\text { Case B: } \\
\text { (short) }\end{array}$ & 62.70 & 63.48 & 52.10 & 51.40 \\
$\begin{array}{c}\text { Case C: } \\
(50 \text { Ohm) }\end{array}$ & 60.44 & 61.90 & 46.93 & 47.10 \\
\hline \hline
\end{tabular}

Note: The transistor's input power remains as $6.5 \mathrm{dBm}$ at $2 \mathrm{GHz}$.

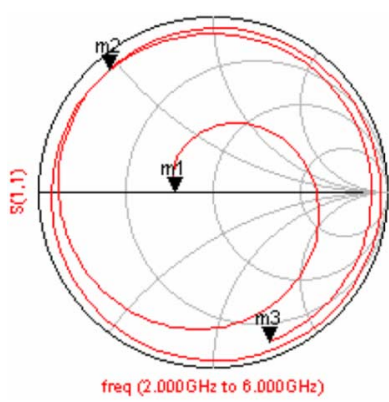

(a)

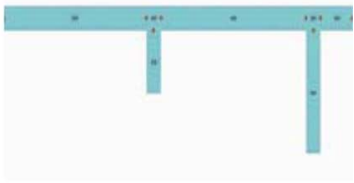

(b)
Fig. 5. Output matching network to supply the optimal reflection coefficients predicted by RT-ALP: (a) S parameter at $f_{0}, 2 f_{0}$ and $3 f_{0}$ frequency (marker 1,2 , and 3 , respectively) and (b) layout (left end to transistor and right end to $50 \Omega)$.

Table I lists the three typical cases (A, B, C) of the PAE contours in Fig. 4 selected for comparison. In each case both the PAE and output power results from the RT-ALP are consistent with those from the constant phase measurement. The small difference indicates that memory effects are small for the $200 \mathrm{kHz}$ RT-ALP modulation frequency used.

With the RT-ALP tuning at $3 f_{0}$, it was found that the performance improvement from controlling the third harmonic is almost insignificant for this particular device. The resulting optimal load terminations for the output matching are then: $\Gamma_{L}\left(\omega_{0}, t\right)=$ $-0.2, \Gamma_{L}\left(2 \omega_{0}, t\right)=0.6+j 0.7, \Gamma_{L}\left(3 \omega_{0}, t\right)=0.30-j 0.85$.

Fig. 5 shows the designed output matching network with microstrip substrate of Duroid $\left(\varepsilon_{r}=2.2, H=31 \mathrm{mil}\right)$. The source 


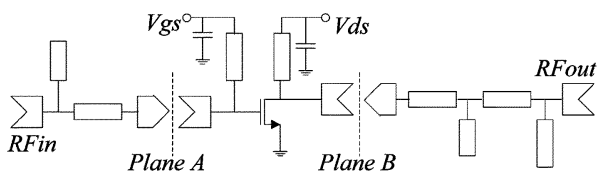

Fig. 6. Simplified schematic of the designed pHEMT PA including bias tees.

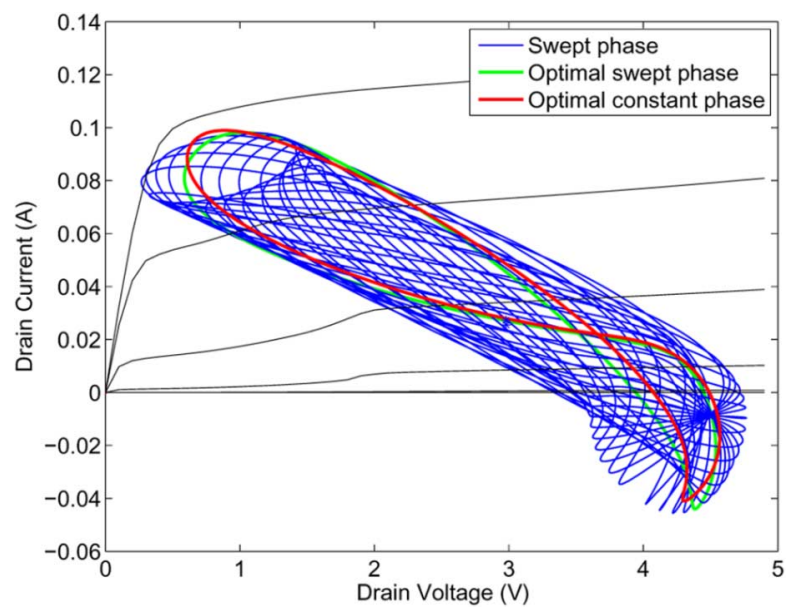

Fig. 7. RF load lines of the transistor for swept phase (blue) with the optimal one (green) and the final constant phase load line(red).

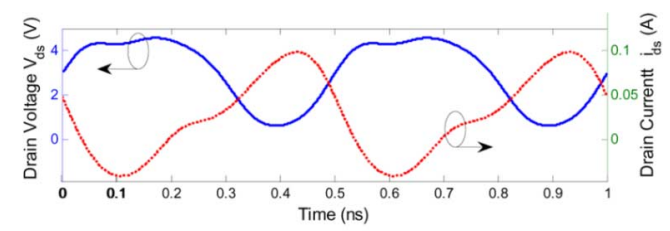

(a)

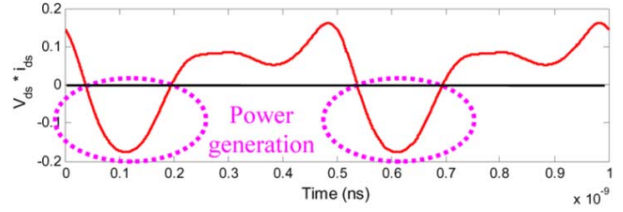

(b)

Fig. 8. (a) Drain current and voltage waveforms and (b) instantaneous power dissipation at the output: the efficiency is optimized when the difference between positive and negative areas is the smallest fraction of the DC power dissipated.

matching network is a conjugate match of the input impedance. The fabricated amplifier with bias tees (Fig. 6) is measured with the reference planes de-embedded to the planes $\mathrm{A}$ and $\mathrm{B}$ given in Fig. 6. Under the input power of $6.5 \mathrm{dBm}$ at $2 \mathrm{GHz}$, the PA achieves PAE of $68.5 \%$ with $64.1 \mathrm{~mW}$ fundamental output power and $15.9 \mathrm{~dB}$ gain. The PAE and Pout results are very close to the findings in Table I, revealing the reliability of the RT-ALP design methodology.

The associated RF loadline is shown in Fig. 7. Note that this is the orthogonality of the time-domain drain voltage and current waveforms in Fig. 8(a) with power generation and absorption in Fig. 8(b) balancing which yields the high PAE.

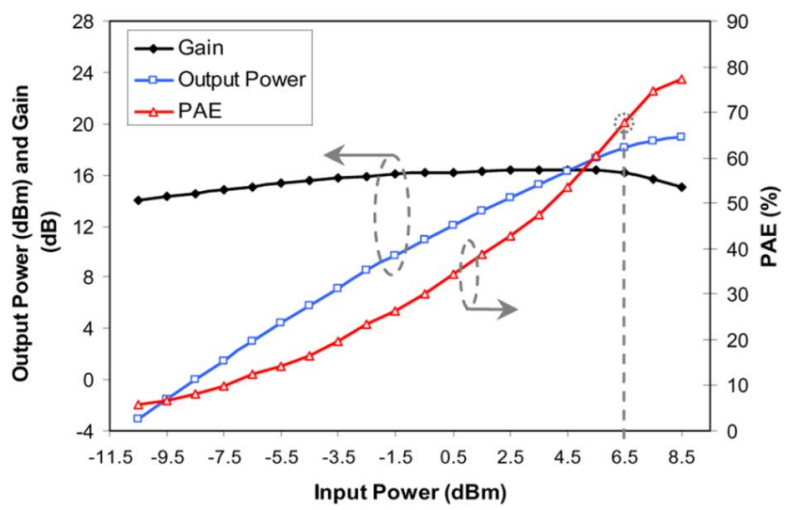

Fig. 9. Pout, Gain, and PAE versus the input power sweep at $2 \mathrm{GHz}$.

From the power sweep measurement in Fig. 9, we can observe a PAE of $68.5 \%$ with $18 \mathrm{dBm}$ output power and $16 \mathrm{~dB}$ gain at $2 \mathrm{GHz}$. Below the $1 \mathrm{~dB}$ compression point, this PA achieves a PAE up to $74.6 \%$ with $18.6 \mathrm{dBm}$ output power.

\section{CONCLUSION}

We have presented the design of a high efficiency p-HEMT RF PA using a novel multiharmonic RT-ALP. The methodology enables to efficiently investigate the optimal load mismatches at the fundamental and harmonics to achieve high PAE in a shortened design cycle. A multiharmonic matching network was then designed to realize the p-HEMT PA. The measurement result of $68.5 \%$ PAE at $2 \mathrm{GHz}$ is in good agreement (around $3 \%$ difference) with the RT-ALP prediction. This demonstrates the efficacy of real-time tuning for the interactive design of power efficient PAs.

\section{REFERENCES}

[1] S. C. Cripps, $R F$ Power Amplifiers for Wireless Communications. Norwood, MA: Artech House, 1999.

[2] F. H. Raab, "Class-F power amplifiers with maximally flat waveforms," IEEE Trans. Microw. Theory Tech., vol. 45, no. 11, pp. 2007-2012, Nov. 1997.

[3] S. Gao, "High-efficiency class F RF/microwave power amplifiers," IEEE Microw. Mag., vol. 7, pp. 40-48, Feb. 2006.

[4] R. B. Stancliff and D. D. Poulin, "Harmonic load-pull," in IEEE MTT-S Int. Dig., Apr. 1979, pp. 185-187.

[5] G. P. Bava, U. Pisani, and V. Pozzolo, "Active load technique for loadpull characterization at microwave frequencies," Electron. Lett., vol. 18, pp. 178-180, Feb. 1982.

[6] D. Barataud, F. Blache, A. Mallet, P. P. Bouysse, J. Nebus, J. P. Villotte, J. Obregon, J. Verspecht, and P. Auxemery, "Measurement and control of current/voltage waveforms of microwave transistors using a harmonic load-pull system for the optimum design of high efficiency power amplifiers," IEEE Trans. Instrum. Meas., vol. 48, no. 4, pp. 835-842, Aug. 1999.

[7] F. Verbeyst and M. V. Bossche, "Real-time and optimal PA characterization speeds up PA design," in Proc. 34th Eur. Microw. Conf., Amsterdam, The Netherlands, 2004, pp. 431-434.

[8] X. Cui, S. J. Doo, P. Roblin, G. H. Jessen, R. G. Rojas, and J. Strahler, "Real-time active load-pull of the 2 nd \& 3rd harmonics for interactive design of non-linear power amplifiers," in Proc. 68th ARFTG, Dec. 2006, pp. 42-49. 